\title{
Avaliação de técnicas de biópsia renal em ovinos ${ }^{1}$
}

\author{
Pedro B. Néspoli2*, Valentim A. Gheller ${ }^{3}$, Paulo V. Peixoto ${ }^{4}$, Antônio U. \\ Carvalho $^{3}$, Ticiana N. França ${ }^{5}$, Elias J. Facury Filho ${ }^{3}$, Douglas K. Godoy de \\ Araújo $^{6}$ e Angela I. Bordin ${ }^{7}$
}

\begin{abstract}
Néspoli P.B., Gheller V.A., Peixoto P.V., Carvalho A.U., França T.N., Facury Filho E.J., Godoy de Araújo D.K. \& Bordin A.I. 2010. [Evaluation of renal biopsy techniques in sheep.] Avaliação de técnicas de biópsia renal em ovinos. Pesquisa Veterinária Brasileira 30(3):260-266. Departamento de Clínica Médica Veterinária, Universidade Federal de Mato Grosso do Sul, Av. Fernando Corrêa da Costa s/n, Coxipó, Cuiabá, MT 78060900, Brazil. E-mail: nespoli@ufmt.br

Due to lack of studies about renal biopsies in sheep, a comparative study was performed for three renal biopsy techniques in this species. In this study, nine crossbred (Santa Inês) ewe lamb $(26.64 \mathrm{~kg} \pm 4,86)$ were used in serial procedures with one week consecutive intervals. The clinical aspects, clinical pathological findings, renal sample weights, histology quality, number of glomeruli, and the presence of artifacts in the histology slices were evaluated using the techniques of percutaneous blind biopsy, ultrasound guided biopsies and of videolaparoscopic procedure. No relevant hematological or biochemical alterations were observed in the animals subjected to renal biopsies and the clinical manifestations detected were slight and transitory, except for one case of urethral obstruction by blood clot. The blind technique was related to more frequent and severe cases of hematuria, with more severe damage to the renal tissue and to the only case of obstruction of the urinary flow. In the videolaparocopic technique, the average weight of the samples was superior and hematuria was slight and transitory. A direct relation was seen between occurrence of severe hematuria and presence of transitional epithelium in the samples and the number of trials used for obtainment of fragments.
\end{abstract}

INDEX TERMS: Renal biopsy, videolaparoscopy, ultrasonography, sheep.

RESUMO.- Devido à escassez de trabalhos sobre biópsias renais em ovinos foi desenvolvido um estudo comparativo entre três técnicas de biópsia renal nesta espécie.

\footnotetext{
${ }^{1}$ Recebido em 11 de setembro de 2009.

Aceito para publicação em 13 de dezembro de 2009.

2 Departamento de Clínica Médica Veterinária, Faculdade Agronomia e Medicina Veterinária, Universidade Federal de Mato Grosso do Sul (UFMT), Av. Fernando Corrêa da Costa s/n, Coxipó, Cuiabá, MT 78060-900, Brasil. *Autor para correspondência: nespoli@ufmt.br

${ }^{3}$ Departamento de Patologia e Clínica Veterinárias, Escola de Veterinária, Universidade Federal de Minas Gerais (UFMG), Av. Antônio Carlos 6627, caixa postal 567, Belo Horizonte, MG 30123-970, Brasil.

${ }^{4}$ Departamento de Nutrição Animal e Pastagem, Instituto de Zootecnia, Universidade Federal Rural do Rio de Janeiro (UFRRJ), Seropédica, RJ 23890-000, Brasil.

${ }^{5}$ Departamento de Epidemiologia e Saúde Pública, Instituto de Veterinária, UFRRJ, Seropédica, RJ.

${ }^{6}$ Graduando em Medicina Veterinária, Escola de Veterinária, UFMG, Belo Horizonte, MG..

${ }^{7}$ Programa de Doutorado em Ciência Animal, Escola de Veterinária, UFMG, Belo Horizonte, MG.
}

Neste estudo foram utilizadas nove ovelhas $(26,64 \mathrm{~kg}$ $\pm 4,86$ ) mestiças (Santa Inês) em procedimentos seriados, com intervalos consecutivos de uma semana. Foram avaliados os aspectos clínicos, achados de patologia clínica, o peso das amostras renais, a qualidade histológica, o número de glomérulos e a presença de artefatos no corte histológico da técnica de biópsia percutânea cega, da biópsia guiada por ultrassonografia e do procedimento videolaparoscópico. Não foram observadas alterações hematológicas ou bioquímicas relevantes nos animais submetidos às biópsias renais e as manifestações clínicas detectadas foram leves e transitórias, exceto por um caso de obstrução uretral por coágulo sangüíneo. A técnica percutânea cega foi relacionada à maior ocorrência e gravidade de hematúria, com danos mais graves ao tecido renal e com o único caso de obstrução do fluxo urinário. Na técnica videolaparoscópica, o peso médio das amostras foi superior e a hematúria discreta e transitória. Verificou-se relação direta entre a ocorrência de hematú- 
ria grave e a presença de epitélio de transição nas amostras e o número de tentativas utilizado para a obtenção dos fragmentos.

TERMOS DE INDEXAÇÃO: Ovinos, biópsia renal, videolaparoscopia, ultrassonografia, ovinos.

\section{INTRODUÇÃO}

A biópsia renal tem sido estudada apenas de forma superficial na espécie ovina. Embora o seu potencial para o diagnóstico de afecções renais seja pouco útil nessa espécie, em virtude da baixa incidência destas doenças, o uso de biópsias seriadas é bastante promissor nos estudos de farmacocinética (Toutain et al. 1985) e de toxicologia. Entretanto, os trabalhos disponíveis se limitam a poucos estudos experimentais invasivos e pouco práticos, nos quais foram utilizadas metodologias cirúrgicas (Mitchell \& Williams 1975) ou biópsias seriadas percutâneas após translocação cirúrgica de ambos os rins para o tecido subcutâneo (Toutain et al. 1985, Brown \& Baird 1988). Não há referência do uso de métodos mais recentes descritos em outras espécies como as biópsias renais por videolaparoscopia (Chiesa et al. 2006) ou guiadas por ultrassonografia (Wisloof et al. 2003), nem tampouco estudos que comparem as diferentes técnicas de biópsia renal.

Considerando o exposto, esse trabalho foi desenvolvido com o objetivo de se compararem três técnicas de biópsias renais, exeqüíveis em condições de campo, frente à obtenção das amostras e à ocorrência de complicações e de efeitos adversos. Foram descritas e comparadas uma técnica videolaparoscópica, uma técnica percutânea cega e uma percutânea guiada por ultrassonografia.

\section{MATERIAL E MÉTODOS}

\section{Animais e procedimentos experimentais}

Foram utilizadas nove borregas (6 Santa Inês e 3 Santa Inês X Texel), com idade entre 6 e 18 meses, e peso médio de $26,64 \mathrm{~kg} \pm 4,86$. Os animais foram alocados no Hospital Veterinário, UFMG, alimentados com capim elefante, feno de Tifton e ração comercial, vermifugados e pesados rotineiramente em intervalos semanais. Após a conclusão do experimento os animais foram submetidos à eutanásia, de acordo com protocolo no 122/2007, CETEA-UFMG. Cada animal foi submetido a todas as três técnicas de biópsia e a ordem de execução foi definida por sorteio. Todas as técnicas foram realizadas sobre o rim direito, em intervalos semanais. Em cada procedimento, as biópsias foram repetidas até se obter um fragmento de tecido com tamanho adequado para avaliação histológica.

Os animais foram submetidos a jejum alimentar de 12 horas, medicados com cloridrato de xilazina (Rompum $\left.{ }^{\circledR}\right)^{8}(0,10 \mathrm{mg} /$ $\mathrm{kg}$ ) e contidos em decúbito lateral esquerdo. Após tricotomia e anti-sepsia foram realizadas infiltrações com $5 \mathrm{ml}$ de cloridrato de lidocaína $2 \%$ (Anestésico L Pearson $\left.{ }^{\circledR}\right)^{9}$ nas linhas de incisão e nos pontos de introdução das agulhas de biópsia (Fig.1). Durante o procedimento os animais receberam $500 \mathrm{ml}$ de solução fisiológica IV e 20mg/animal de furosemida a 1\% (Furosefarma) $)^{10}$.

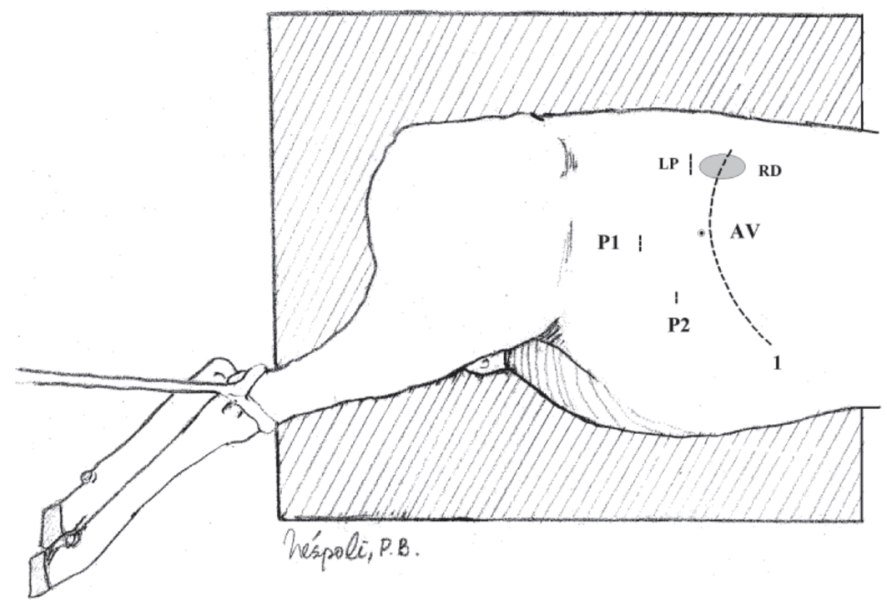

Fig.1. Locais de estabelecimento dos portais para videolaparoscopia, introdução da agulha de Veress e laparotomia. (1) Margem caudal do gradil costal; (AV) Local para introdução da agulha de Veress; (P1) Local do primeiro portal para introdução do endoscópio; (P2) Segundo portal para introdução de instrumentais; (LP) Local da laparotomia para realização da biópsia cega. (RD) Rim direito.

\section{Técnicas de biópsia renal}

Biópsia renal percutânea cega (BC). Após realização de incisão sobre a parede abdominal, perpendicular ao processo transverso da terceira vértebra lombar (Fig.1), o pólo caudal do rim direito foi localizado e fixado manualmente. Na seqüência, uma agulha Tru-cut semi-automática (Bloodline $\left.{ }^{\circledR}\right)^{11}$ foi introduzida na parede abdominal através de pequena incisão de pele cranial à primeira. A haste obturadora interna da agulha foi avançada perpendicularmente no pólo caudal do rim. Após a manipulação, a agulha foi retirada da cavidade abdominal, o fragmento de tecido renal recolhido e a parede abdominal suturada de acordo com os preceitos cirúrgicos.

Biópsia renal percutânea guiada por ultrassonografia (BU). A introdução e a manipulação da agulha Tru-cut semiautomática no rim direito foi monitorada através de imagens ultrassonográficas, obtidas com o aparelho de ultrassonografia

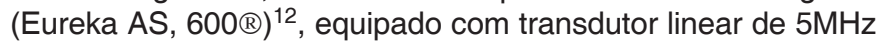
sem guia para a passagem da agulha de biópsia.

Biópsia renal por videolaparoscopia com pinça de Blakesley (BL). Após a preparação do animal, desinfecção do instrumental de endoscopia (Karl Storz $\left.{ }^{\circledR}\right)^{13}$ e anestesia local, a agulha de Veress foi introduzida na cavidade abdominal na porção dorsocranial do flanco direito, logo atrás da última costela (Fig.1). Através desta agulha o fluxo de $\mathrm{CO}_{2}$ foi controlado automaticamente e a pressão intra-abdominal mantida em torno de $10 \mathrm{~mm}$ de $\mathrm{Hg}$. O conjunto trocarte/cânula de $11 \mathrm{~mm}$ foi

\footnotetext{
${ }^{8}$ Bayer S.A., Fábrica de Belford Roxo, Estrada Boa Esperança 650, Belford Roxo, Rio de Janeiro, RJ 26110-100.

${ }^{9}$ Eurofarma Laboratórios Ltda, Rua Cubatão 929, São Paulo, SP.

${ }^{10}$ Farmace Ind. Química Farmacêutica Cearense Ltda, Rodov. Dr. Antônio Líro Callou, Km 2, Barabalha, CE.

${ }^{11}$ Bloodline S.p.A,. Via Statale 12, n.149/A, 41036 Medolla (MO), Italy.

12 Medison Co., Discusser and Medison Building 1003, Daechi-dong Gangnam-gu, Seoul 135-280, Korea.

${ }^{13}$ Karl Storz GmbH \& Co.KG. Mittelstr.8, D-78532 Tuttlingen, Alemanha.
} 

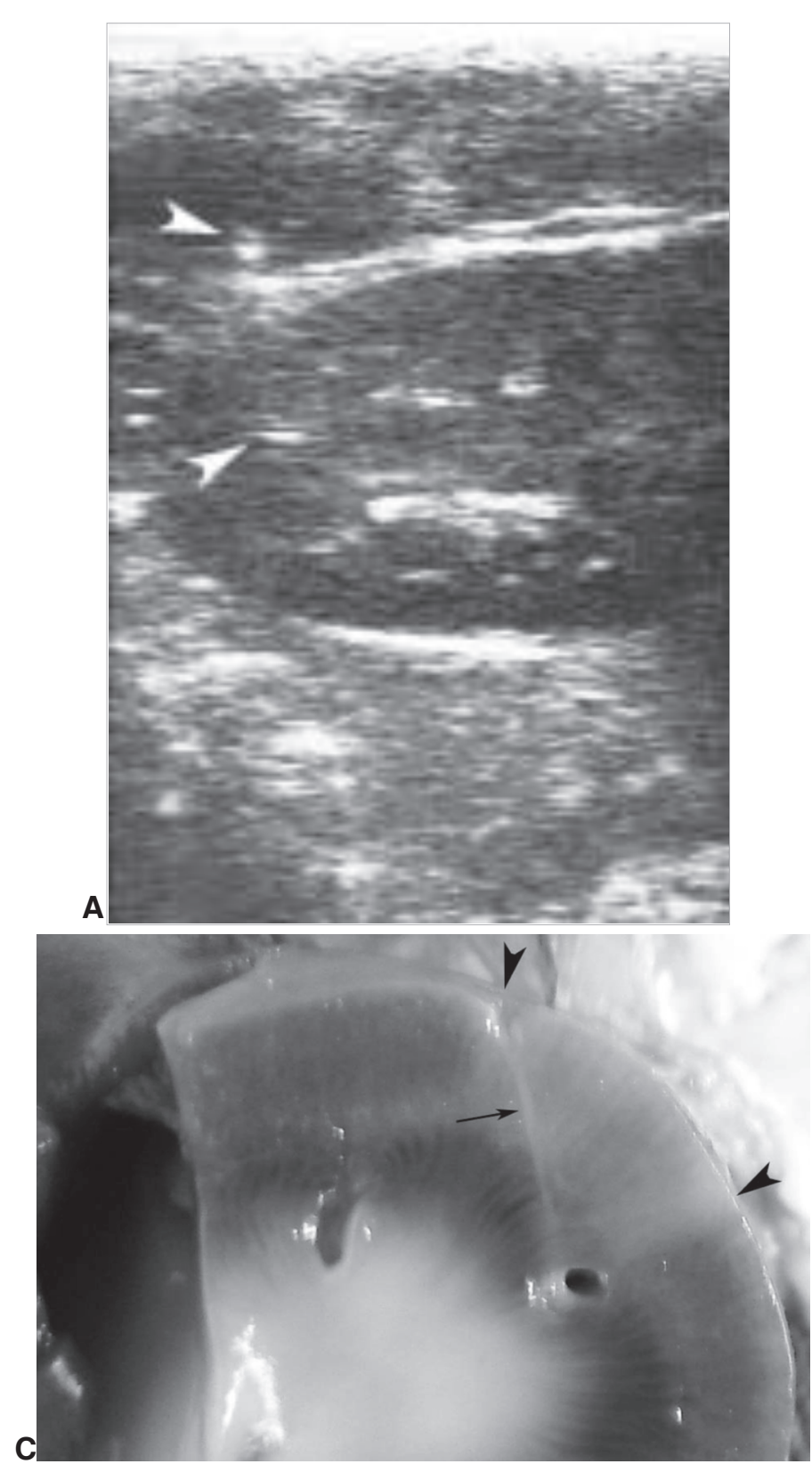

introduzido no abdome através de incisão de pele para estabelecer o portal 1 (P1) e um conjunto trocarte/cânula de $6 \mathrm{~mm}$ para estabelecer o P2 (Fig.1). Após inserção do endoscópio rígido na cavidade abdominal através do P1, uma tesoura cirúrgica foi introduzida pelo P2 para se proceder a incisão do peritônio sobre a superfície renal. Na seqüência, a pinça de Blakesley foi introduzida através do mesmo portal para a retirada de fragmento do córtex renal (Fig.2D).

\section{Processamento do material}

Os fragmentos renais obtidos através das biópsias, independente do número de tentativas realizadas, foram fixados em formol a $10 \%$ em um único recipiente, pesados e enviados para o Setor de Anatomia Patológica, Convênio Universidade Federal Rural do Rio de Janeiro/Embrapa. Esses fragmentos foram processados rotineiramente para estudo histológico e corados pela Hematoxilina e Eosina (HE). Foram analisadas a
Fig.2. (A) Imagem ultrassonográfica do trajeto da agulha de biópsia no córtex renal (cabeças de seta). (B) Apreensão do tecido renal com a pinça de Blakesley abaixo do peritônio. (C) Corte do rim sobre depressão localizada da superfície renal evidencia trajeto fibroso (seta) da biópsia com agulha; no córtex adjacente verifica-se uma área típica de infarto renal (cabeças de seta). (D) Lesão renal determinada pelas biópsias renais. Áreas do córtex renal mais delgadas ou ausentes; medular adjacente apresenta-se retraída e substituída por tecido conjuntivo
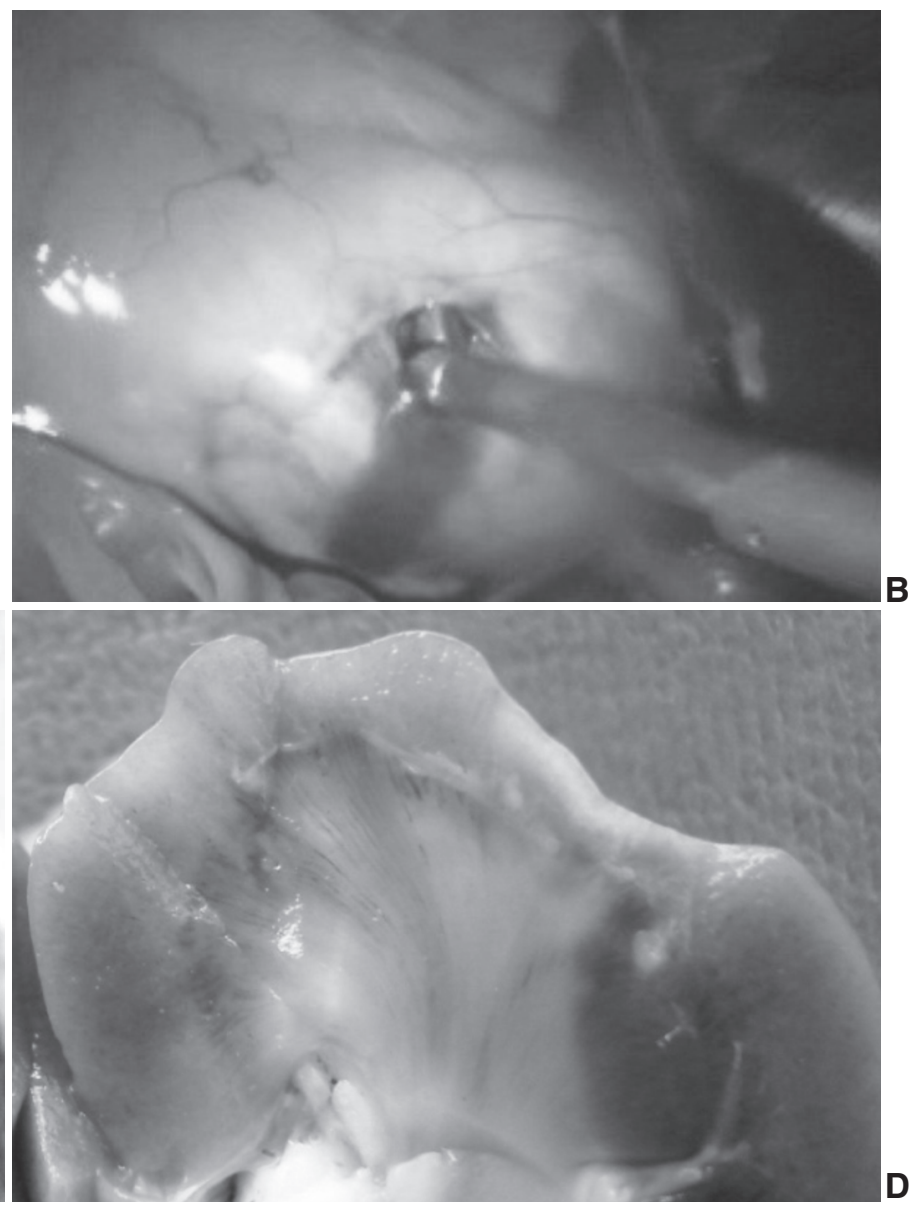

qualidade e a presença de artefatos nas amostras, o número de glomérulos, a quantidade de arteríolas arciformes e a presença de tecido medular e de epitélio de transição no tecido.

\section{Exame clínico e patologia clínica}

O exame clínico diário foi realizado de acordo com a metodologia de exame geral descrita por Stöber (1993). No dia do procedimento os ovinos foram medicados com dose única de tetraciclina (Terramicina $L A ®)^{14}(20 \mathrm{mg} / \mathrm{kg})$ e de flunixin meglumine (Flunamine $\mathbb{R})^{8}(1,1 \mathrm{mg} / \mathrm{kg})$. A hematimetria foi realizada através de contagem automática de células antes do início do experimento e seis dias após a realização das técnicas de biópsia. Os exames da uréia e creatinina foram realizados um dia antes e em três dias consecutivos após cada procedimento através de espectrofotometria. A presença de

\footnotetext{
14 Pfizer, Av. Monteiro Lobato 2270, Guarulhos, SP 07190001.
} 
Quadro 1. Observação de hematúria em ovinos de acordo com a técnica de biópsia, o número de tentativas e avaliação histológica das amostras renais

\begin{tabular}{|c|c|c|c|c|c|c|c|c|c|c|c|c|}
\hline \multicolumn{3}{|c|}{ Execução das técnicas } & \multicolumn{3}{|c|}{ Avaliação histológica da amostra } & \multirow{2}{*}{\multicolumn{7}{|c|}{$\begin{array}{l}\text { Hematúria no período imediato à biópsia }(0) \text { e nos dias } \\
\text { subseqüentes }\end{array}$}} \\
\hline \multirow[t]{2}{*}{ Ovinos } & \multirow{2}{*}{$\begin{array}{l}\text { Técnica } \\
\text { biópsia }^{a}\end{array}$} & \multirow[t]{2}{*}{ Tentativas } & \multirow{2}{*}{$\begin{array}{c}\text { Tecido } \\
\text { medular }\end{array}$} & \multirow{2}{*}{$\begin{array}{l}\text { Tecido da } \\
\text { pelve renal }\end{array}$} & \multirow{2}{*}{$\begin{array}{l}\text { Arteríolas } \\
\text { arciformes }\end{array}$} & & & & & & & \\
\hline & & & & & & 0 & 1 & 2 & 3 & 4 & 5 & 6 \\
\hline \multirow[t]{3}{*}{1} & $\mathrm{BU}$ & 2 & - & h & 3 & - & - & - & & & & \\
\hline & $\mathrm{BC}$ & 2 & +++ & - & 1 & $\mathrm{H}$ & $+t^{\mathrm{b}}$ & ++ & + & - & - & \\
\hline & $\mathrm{BL}$ & 1 & + & - & 1 & + & - & - & & & & \\
\hline \multirow[t]{4}{*}{2} & $\mathrm{BU}$ & 1 & $+(+)$ & - & - & - & - & - & & & & \\
\hline & $\mathrm{BL}$ & 1 & - & - & - & + & - & - & & & & \\
\hline & $\mathrm{BC}$ & 3 & + & Presente & 1 & ++ & $\mathrm{H}^{f}$ & $\mathrm{H}$ & ++ & + & + & +9 \\
\hline & $\mathrm{BC}$ & 1 & + & - & 1 & $++/+++{ }^{C}$ & - & - & & & & \\
\hline \multirow[t]{2}{*}{3} & $\mathrm{BL}$ & 1 & $++(+)$ & - & 1 & & & - & & & & \\
\hline & $\mathrm{BU}$ & 1 & - & - & - & - & - & - & & & & \\
\hline \multirow[t]{3}{*}{4} & $\mathrm{BC}$ & 1 & - & - & - & - & - & - & & & & \\
\hline & $\mathrm{BU}$ & 2 & - & - & - & - & - & - & & & & \\
\hline & $\mathrm{BL}$ & 1 & - & - & - & - & - & - & & & & \\
\hline \multirow[t]{3}{*}{5} & $\mathrm{BC}$ & 1 & - & - & - & +/++ & & - & & & & \\
\hline & $\mathrm{BU}$ & 2 & - & - & - & - & - & - & & & & \\
\hline & $\mathrm{BL}$ & 1 & - & - & - & - & - & - & & & & \\
\hline \multirow[t]{3}{*}{6} & $\mathrm{BL}$ & 1 & + & - & 3 & + & - & - & & & & \\
\hline & $\mathrm{BC}$ & 1 & - & - & - & - & - & - & & & & \\
\hline & $\mathrm{BU}$ & 2 & - & - & - & - & - & - & & & & \\
\hline \multirow[t]{3}{*}{7} & $\mathrm{BL}$ & 1 & ++ & - & 2 & ++ & - & - & & & & \\
\hline & $\mathrm{BC}$ & 1 & ++ & - & - & - & - & - & & & & \\
\hline & $\mathrm{BU}$ & 3 & +++ & - & 3 & - & $\mathrm{NR}^{\mathrm{e}}$ & - & & & & \\
\hline \multirow[t]{3}{*}{8} & $\mathrm{BU}$ & 2 & ++ & Presente & - & $H^{d}$ & ++ & ++ & + & + & - & - \\
\hline & $\mathrm{BL}$ & 1 & - & - & - & - & - & - & & & & \\
\hline & $\mathrm{BC}$ & 2 & + & Presente & - & $++/+++$ & + & ++ & - & - & & \\
\hline \multirow[t]{3}{*}{9} & $\mathrm{BL}$ & 2 & + & - & 2 & & - & - & & & & \\
\hline & $\mathrm{BU}$ & 2 & - & - & - & - & - & - & & & & \\
\hline & $\mathrm{BC}$ & 3 & - & - & - & $\mathrm{H}$ & ++ & ++ & - & - & & \\
\hline
\end{tabular}

a Técnicas de biópsia renal dispostas na coluna de acordo com a ordem de execução. $\mathrm{BC}=$ Biópsia percutânea cega. $\mathrm{BU}=\mathrm{Biópsia}$ guiada por ultrassonografia. $\mathrm{BL}=$ Biópsia com pinça laparoscópica. ${ }^{\mathrm{b}}$ Hematúria microscópica: + (5-10 hemácias por ml); ++ (50 hemácias por $\mathrm{ml}) ;+++(300$ hemácias por $\mathrm{ml}) .{ }^{c}$ Presença de dois pequenos coágulos sanguíneos na urina com aspecto macroscópico normal. d Hematúria macroscópica. Neste animal a urina apresentava coloração avermelhada intensa com presença de grande coágulo sanguíneo. ${ }^{e}$ Exame não realizado. ${ }^{f}$ Urina com coloração marrom escura. ${ }^{9}$ Apresentou hematúria microscópica (+) por mais três dias (D7-D9). A hematúria cessou nos dias subseqüentes (D10 e D11). ${ }^{\mathrm{h}}$ Ausentes.

hematúria foi aferida através de fita para urinálise ${ }^{15}$. Naqueles animais positivos, o exame foi repetido até a remissão deste sinal por dois dias consecutivos.

Os dados paramétricos foram comparados através do teste t de Student (Sampaio 2002) e os não paramétricos ou não normais através da aplicação do programa Bioestat 4.0 (Ayres et al. 2005).

\section{RESULTADOS}

Durante o experimento o peso dos ovinos permaneceu estável e as poucas manifestações clínicas observadas (3/27) foram leves, transitórias e caracterizadas por leve hipertermia, congestão discreta das mucosas e leve diminuição dos movimentos ruminais. Apenas um animal apresentou complicações clínicas significativas. Um dia após a realização da biópsia cega, o animal dois demonstrou sinais clínicos típicos de obstrução uretral. A sondagem uretral revelou uma obstrução por coágulo sanguíneo e grande volume de urina marrom escura. Imediatamente após a sondagem, o animal apresentou apetite e remissão dos sinais clínicos.

15 Dialab diagnósticos S/A, Av. do Contorno 2090, sala 701/702, Floresta, Belo Horizonte, MG 30110-070.
Verificou-se elevação da uréia na faixa de 100 e 120mg/ dl, por dois dias consecutivos em um e níveis transitórios acima de $60 \mathrm{mg} / \mathrm{dl}$ em outros dois ovinos. Nos demais animais os níveis de uréia oscilaram entre 20 e $60 \mathrm{mg} / \mathrm{dl}$. Os valores de creatinina, por outro lado, só se elevaram acima de $2 \mathrm{mg} / \mathrm{dl}$ em dois casos (Ovinos 1 e 7) em que houve desidratação não relacionada com os procedimentos de biópsia.

O número médio de eritrócitos oscilou entre12 e $14\left(\mathrm{X} 10^{6} / \mathrm{ml}\right)$ e os valores médios do hematócrito entre 30 e 33\%. Apesar da contagem média de plaquetas ter permanecido entre 300 e $400\left(\times 10^{3} / \mathrm{ml}\right)$, na maior parte do experimento, o número médio da última aferição foi levemente maior que $200\left(\times 10^{3} / \mathrm{ml}\right)$. Do total de 27 biópsias renais realizadas houve hematúria em 11 procedimentos. Quatro foram macroscópicas e sete foram microscópicas (Quadro 1). Observou-se que a presença de epitélio de transição na amostra esteve invariavelmente relacionada com a ocorrência e a intensidade dessas hematúrias. Nas três amostras em que se recuperou esse tecido houve hematúria macroscópica em duas circunstâncias e hematúria microscópica intensa em outra.

A perfuração da bolsa omental foi a principal dificulda- 
de encontrada durante os procedimentos de videolaparoscopia e a identificação do trajeto da agulha de biópsia o principal fator complicador das biópsias guiadas por ultrassonografia. As hemorragias observadas no presente estudo com uso da pinça laparoscópica foram excessivas em apenas uma ocasião em que houve necessidade de compressão do ponto de biópsia com a pinça laparoscópica.

Na maior parte dos animais os achados macroscópicos atribuíveis às técnicas percutâneas se limitaram a trajetos fibrosos lineares transversais do parênquima, associadas às eventuais áreas de infarto cortical (Fig.2C). Porém, dois animais apresentaram lesões mais amplas da superfície cortical, com e sem envolvimento medular. O Ovino 2 demonstrou diminuição do volume renal, achatamento e depressão irregular do pólo caudal; necrose cortical e fibrose do tecido medular adjacente (Fig.2D). As lesões provocadas pelo uso da pinça laparoscópica caracterizavam-se por depressões localizadas, limitadas ao córtex renal e associadas a aderências localizadas da cápsula renal e do tecido adiposo peri-renal.

O número de tentativas despendidas, os pesos e o número de glomérulos das amostras renais estão dispostos nos Quadros 2 e 3. A qualidade das amostras recupe-

Quadro 2. Comparação do número médio de tentativas despendidas para obtenção de amostras representativas, do peso médio das amostras $(\mathrm{mg})$ e do número de glomérulos obtidos com as técnicas de biópsia renal

\begin{tabular}{lccc}
\hline & $\mathrm{BC}^{1}$ & $\mathrm{BU}^{2}$ & $\mathrm{BL}^{3}$ \\
\hline № de tentativas & $41,67^{\mathrm{ab}}$ & $1,89^{\mathrm{a}}$ & $1,11^{\mathrm{b}}$ \\
Peso total & $17,83^{\mathrm{a}}$ & $12,06^{\mathrm{a}}$ & $91,3^{\mathrm{b}}$ \\
Peso/ tentativas & $13,01^{\mathrm{a}}$ & $7,27^{\mathrm{a}}$ & $85,61^{\mathrm{b}}$ \\
Glomérulos totais & $36.78^{\mathrm{a}}$ & $26.22^{\mathrm{a}}$ & $>100^{\mathrm{b}}$ \\
Glomérulos/tentativas & $30,37^{\mathrm{a}}$ & $15,91^{\mathrm{a}}$ & $>100^{\mathrm{b}}$
\end{tabular}

${ }^{1}$ Biópsia percutânea cega; ${ }^{2}$ biópsia guiada por ultrassonografia; ${ }^{3}$ biópsia com pinça laparoscópica; ${ }^{4}$ Médias com letras iguais na mesma linha não diferem significativamente $(p<0,05)$.

Quadro 3. Intervalo de variação do peso das amostras $(\mathrm{mg})$ renais obtidas com apenas uma tentativa

\begin{tabular}{lccc}
\hline & $\mathrm{BC}^{1}$ & $\mathrm{BU}^{2}$ & $\mathrm{BL}^{3}$ \\
\hline Variação do peso & 10,8 a 21,7 & 11,7 a 14,6 & 48 a 116,8 \\
Variação no de glomérulos & 16 a 72 & 12 a 44 & 9 a > 100 \\
Biópsias comuma tentativa & 5 & 2 & 8
\end{tabular}

${ }^{1}$ Biópsia percutânea cega; ${ }^{2}$ biópsia guiada por ultrassonografia; ${ }^{3}$ biópsia com pinça laparoscópica.

radas com pinça laparoscópica foi levemente superior ao daquelas obtidas com as técnicas percutâneas, muito embora os fragmentos obtidos com as diferentes técnicas foram considerados regulares ou bons na maior parte das avaliações. Não foram identificadas diferenças relevantes sobre o grau de fragmentação parcial das amostras, a presença de hemorragia e de tecido medular no tecido recuperado.

\section{DISCUSSÃO E CONCLUSÕES}

\section{Alterações clínicas e laboratoriais associadas aos pro- cedimentos}

Verificou-se que a biópsia renal seriada pode ser conduzida com poucas complicações pós-cirúrgicas. Em apenas uma circunstância houve complicação significativa, com obstrução do fluxo urinário por coágulo sangüíneo (Ovino 2). Casos de obstrução uretral como esse não têm sido descritos na literatura consultada e os relatos disponíveis incluem principalmente a obstrução da pelve renal (Jeraj et al. 1982, Osborne et al. 1996). Para prevenir essas formações de coágulos tem sido preconizada a administração de solução fisiológica antes e durante o procedimento (Jeraj et al. 1982). Embora a mesma quantidade de fluido descrita em estudos de caprinos (Wisloff et al. 2003) tenha sido utilizada nesse estudo, a administração de $500 \mathrm{ml}$ de soro fisiológico e de furosemida não se mostrou completamente eficaz.

Dentre os estudos de biópsia renal, realizados em animais de produção, são descritos dois casos de morte. Um bovino por hemorragia não corrigível (Chiesa et al. 2006) e um búfalo em função de peritonite (Ramkumar et al. 1972). Em outros artigos correspondentes não houve descrições de complicações clínicas significativas (Garner et al. 1968, Osborne et al. 1968, Mitchell \& Williams 1975, Naoi et al. 1985).

As alterações de uréia e creatinina observadas não tiveram relação com a realização dos procedimentos de biópsia renal. A manutenção dos níveis de uréia acima do nível de referência, 18 a $31 \mathrm{mg} / \mathrm{dl}$ (Meyer et al. 1995), na maioria dos animais avaliados, provavelmente, foi determinada por causas extra-renais. Esta hipótese foi corroborada pela observação correspondente de níveis normais de creatinina, já que a creatinina não sofre as mesmas influências extra-renais relacionadas com o maior aporte de aminoácidos como ocorre com a uréia (Thrall 2007). Da mesma forma, os dados coincidentes entre uréia e creatinina, observados nos ovinos 1 e 7 , indicaram possivelmente uma menor taxa de filtração glomerular determinada por hipovolemia.

Assim como observado em outros estudos de biópsia renal em bovinos (Naoi et al. 1985) e bubalinos (Ramkumar et al. 1972), não houve no presente experimento alterações relevantes de parâmetros hematológicos. Tanto o hematócrito como a contagem de hemácias permaneceram estáveis durante o experimento e dentro dos limites de referência para a espécie, $27-45 \%$ e 9-15(x106/ml), respectivamente.

A ocorrência de um grande número de casos de hematúria foi um achado comumente encontrado nos trabaIhos correspondentes em diversas espécies (Nash et al. 1983, Naoi et al. 1985, Brow e Baird 1988, Osborne et al. 1996). Assim como anteriormente descrito (Osborne et al. 1996) a hematúria microscópica ocorreu nesse experimento em um grande número de ocasiões, mas em número menor do que o registrado em outros trabalhos realizados em bovinos (Osborne et al. 1968, Naoi et al. 1985). 
Já a hematúria macroscópica, considerada menos comum, ocorreu em cerca de $14 \%$ dos procedimentos, um número bastante acima dos 3\% relatados por Osborne et al. (1996). Tanto as hematúrias macroscópicas quanto as microscópicas apresentaram caráter auto-limitante, conforme relatado por Osborne et al. (1996), porém a sua presença foi mais prolongada nos casos em que havia alteração macroscópica da urina desde o início.

Embora não tenham sido relatadas em outros artigos (Osborne 1971, Jeraj et al. 1982, Nash et al. 1983), a ocorrência e a gravidade da hematúria estiveram invariavelmente relacionadas com a presença de epitélio de transição na amostra recuperada. Em todas as vezes em que foi identificado tal epitélio houve hematúria macroscópica ou microscópica intensa. Já a presença de tecido medular e de arteríolas arciformes nas amostras, aparentemente, não foi associada de forma direta à ocorrência desse achado, uma vez que não houve hematúria em cerca da metade dos casos em que essas estrututras foram detectadas (Quadro 1).

A associação da técnica percutânea cega com maior gravidade e o número de casos de hematúria provavelmente teve relação com a manipulação renal e com a dificuldade em se manter o curso da agulha distante da região medular e da pelve renal. Já o uso das técnicas guiadas por ultrassonografia e por videolaparoscopia foi aparentemente mais eficiente em evitar a lesão de estruturas mais profundas do parênquima renal. Por outro lado, o número de tentativas para obtenção da amostra também teve relação com a gravidade da hematúria. Nos casos de hematúria mais intensa e prolongada foram utilizadas duas ou três tentativas para obtenção das amostras. Já o uso de uma tentativa esteve relacionado somente com a ocorrência de hematúrias microscópicas, que ficaram restritas ao dia da realização da biópsia.

\section{Complicações causadas pelas técnicas}

$\mathrm{Na}$ grande maioria dos procedimentos, as hemorragias foram de baixa intensidade e nenhuma medida de hemostasia foi utilizada, como descrito por Naoi et al. (1985). Por outro lado, nos estudos conduzidos por Chiesa et al. (2006) os procedimentos de hemostasia não foram empregados apenas em alguns casos. Mesmo assim, o autor relata que um animal apresentou hemorragia severa e morreu 12 horas após o terceiro procedimento.

A ocorrência de fibrose linear, descrita em vários artigos (Osborne et al. 1968, Nash et al. 1983, Brow \& Baird 1988), é resultante do dano direto da passagem das aguIhas percutâneas através do tecido renal. Os infartos verificados nesse estudo, assim como as lesões mais extensas observadas nos animais um e dois, foram provavelmente determinados por danos diretos aos vasos sanguíneos renais. Essa afirmativa se baseia no consenso de que a severidade e extensão do dano renal são proporcionais ao número e ao tamanho dos vasos lesados (Osborne et al. 1996) e é por isso que se preconiza confinar o trajeto da agulha, o máximo possível, à região cortical
(Nash et al. 1983), com o intuito de se evitar as áreas mais vascularizadas, como a junção corticomedular.

\section{Avaliação das amostras}

A baixa taxa de recuperação de amostras com apenas uma tentativa associada à técnica guiada por ultrassonografia aliada aos menores pesos da amostras obtidas, provavelmente, refletiram as dificuldades de execução da técnica de biópsia renal guiada por ultrassonografia. Por outro lado, mesmo que a técnica percutânea cega tenha sido mais trabalhosa e mais invasiva, verificou-se que ela pode ser uma alternativa viável para a realização de biópsia renal em ovinos na ausência de equipamentos como a ultrassonografia e de videolaparoscopia.

Todas as técnicas propiciaram a obtenção de amostras com quantidade de glomérulos superior àquela estimada como necessária para avaliações histopatológicas (Quadro 3) (Hoppe et al. 1986, Wise et al. 1989, LauferAmorim et al. 2002). Entre estas estimativas descritas em estudos de medicina humana, considera-se que a representatividade das amostras renais depende de pelo menos quatro ou sete glomérulos e que o aumento deste número não resultaria em maior eficácia diagnóstica da amostra (Laufer-Amorim et al. 2002). Apesar de não haver estudos veterinários similares, alguns autores consideram igualmente adequadas amostras que apresentem tecido medular e um número mínimo de cinco glomérulos (Hoppe et al. 1986, Wise et al. 1989).

Todavia, não há consenso sobre este critério de representatividade. De acordo com Osborne (1971), as informações contidas nas amostras de tecido não dependem diretamente do número de glomérulos. Exemplifica que em determinadas situações o diagnóstico não pode ser estabelecido mesmo com um número ilimitado de glomérulos, ao passo que em outras circunstâncias podese sugerir ou confirmar diagnósticos com apenas um glomérulo. Além disso, foi demonstrado em um estudo retrospectivo que diagnósticos corretos podem ser realizados a partir de amostras com número reduzido de glomérulos (zero a quatro).

O número de glomérulos obtidos através de biópsias renais percutâneas tem sido relatado em diversos estudos de cães e gatos com o uso de técnicas e instrumentais distintos (Jeraj et al. 1982, Nash et al. 1983, Hoppe et al. 1986, Wise et al. 1989, Laufer-Amorim et al. 2002). As médias encontradas entre os estudos incluem 6,4 (Nash 1986), 9,7 (Nash et al. 1986), 13,8 (Nash et al. 1983), 15,93 e 17,53 glomérulos (Laufer-Amorim et al. 2002).

\section{REFERÊNCIAS}

Ayres M., Ayres M.J., Ayres D.L. \& Santos A.A.S. 2005. Bioestat: aplicações estatísticas nas áreas das ciências biomédicas. Mamirauá, Belém. Disponível em <http://www.mamiraua.org.br> Acesso em 25.9.2007.

Brown S.A. \& Baird A.N. 1988. Evaluation of renal gentamicin depletion kinetic properties in sheep, using serial percutaneous biopsies. Am. J. Vet. Res. 49(12):2056-2059.

Chiesa O.A., Cullison R., Anderson D.E., Moulton K., Galuppo L.D. \& 
Bredow J.V. 2006. Development of a technique for serial bilateral renal biopsy in steers. Can. J. Vet. Res. 70:87-93.

Garner H.E., Thurmon J.C. \& Romack F.E. 1968. Biopsy: Surgical method for obtaining large samples of liver, kidney, and muscle from cattle. Am. J. Vet. Res. 29(12):2407-2409.

Hoppe F.E., Hager D.A., Poulos P.W., Ekman S. \& Lindgren P.G. 1986. Comparison of manual and automatic ultrasound guided biopsy techniques. Vet. Radiol. 27:99-101.

Jeraj K., Osborne C.A. \& Stevens J.B. 1982. Evaluation of renal biopsy in 197 dogs and cats. J. Am. Vet. Med. Assoc. 181(4):367-369.

Laufer-Amorim R., Bandarra E.P., Monteiro L.A.J. \& Moura V.M.B.D. 2002. Avaliação quantitativa e qualitativa da técnica de biopsia renal "de janela" em cães. Ciênc. Anim. Bras. 3(2):41-45.

Meyer D.J., Coles E.H. \& Rich L.J. 1995. Medicina de laboratório veterinária: interpretação e diagnóstico. Roca, São Paulo. 320p.

Mitchell B. \& Williams J.T. 1975. Technique for biopsy of renal cortex in sheep. Vet. Rec. 96(18):405.

Naoi M., Kokue E., Takahashi Y. \& Yasumasa K. 1985. Laparoscopicassisted serial biopsy of the bovine kidney. Am. J. Vet. Res. 46(3):699702.

Nash A.S., Boyd J.S., Minto A.W. \& Wright N.G. 1983. Renal biopsy in the normal cat: An examination of the effects of a single needle biopsy. Res. Vet. Sci. 34(3):347-356.

Osborne C.A. 1971. Clinical avaluation of needle biopsy of the kidney and its complications in the dog and cat. J. Am. Vet. Med. Assoc. 158:1213-1229.
Osborne C.A., Bartges J.W., Polzin D.J., Lulich J.P., Johnston G.R. \& Cox V. 1996. Percutaneous needle biospy of the kidney. Vet. Clin. North Am., Small Anim. Pract. 26(6):1461-1504.

Osborne C.A., Farning M.L., Schultz R.H. \& Perman V. 1968. Percutaneous renal biopsy in the cow and horse. J. Am. Vet. Med. Assoc. 153:563-570.

Ramkumar V., Kwatra M.S., Kalra D.S. \& Tyagi R.P.S. 1972. Open renal biopsy in bovines: An experimental study. Indian J. Anim. Sci. 42:915-918.

Sampaio I.B.M. 2002. Estatística Aplicada à Experimentação Animal. $2^{\mathrm{a}}$ ed. Fundação de Ensino e Pesquisa em Medicina Veterinária e Zootecnia, Belo Horizonte. 265p.

Stöber M. 1993. Identificação, anamnese, regras básicas da técnica de exame clínico geral, p.166-225. In: Rosenberger G. (Ed.), Exame Clínico dos Bovinos. $3^{\mathrm{a}}$ ed. Guanabara Koogan, Rio de Janeiro.

Thrall M.A. 2007. Hematologia e Bioquímica Clínica Veterinária. Roca, São Paulo, p.285-310.

Toutain P.L., Pomyers H., Larrieu G., Periquet B. \& More J. 1985. An in vivo model for pharmacokinetic studies in the kidney. J. Pharmacol. Methods 14(1):1-11.

Wise L.A., Allen T.A. \& Cartwright M. 1989. Comparison of renal biopsy techniques in dogs. J. Am. Vet. Med. Assoc. 195(7):935-939.

Wisloff H., Flaoyen N., Ottesen N. \& Hovig T. 2003. Narthecium ossifragum (L.) Huds. causes kidney damage in goats: Morphologic and functional effects. Vet. Pathol. 40:317-327 